\title{
Inappropriate Waveform
}

National Cancer Institute

\section{Source}

National Cancer Institute. Inappropriate Waveform. NCI Thesaurus. Code C63157.

Failure of the device to generate a correctly-shaped pacing output, e.g., a waveform that is too wide. 